Nova Southeastern University

\title{
The Catalog of Edge-On Disk Galaxies from SDSS. I. The Catalog and the Structural Parameters of Stellar Disks
}

\section{V. Bizyaev}

Apache Point Observatory and New Mexico State University; Moscow State University

Stefan Kautsch

Nova Southeastern University, skautsch@nova.edu

A. V. Mosenkov

Universiteit Gent; Central Astronomical Observatory of RAS; St Petersburg State University

V.P. Reshetnikov

St Petersburg State University; Isaac Newton Institute of Chile

N. Ya. Sotnikova

St Petersburg State University; Isaac Newton Institute of Chile

See next page for additional authors

Follow this and additional works at: https://nsuworks.nova.edu/cnso_chemphys_facarticles

Part of the External Galaxies Commons

\section{NSUWorks Citation}

Bizyaev, D. V., Kautsch, S., Mosenkov, A. V., Reshetnikov, V. P., Sotnikova, N. Y., \& Yablokova, N. V. (2014). The Catalog of Edge-On Disk Galaxies from SDSS. I. The Catalog and the Structural Parameters of Stellar Disks. The Astrophysical Journal, 787, (1), 1 - 12. https://doi.org/10.1088/0004-637X/787/1/24. Retrieved from https://nsuworks.nova.edu/cnso_chemphys_facarticles/107 
Authors

D. V. Bizyaev, Stefan Kautsch, A. V. Mosenkov, V. P. Reshetnikov, N. Ya. Sotnikova, and N. V. Yablokova 


\title{
THE CATALOG OF EDGE-ON DISK GALAXIES FROM SDSS. I. THE CATALOG AND THE STRUCTURAL PARAMETERS OF STELLAR DISKS
}

\author{
D. V. Bizyaev ${ }^{1,2}$, S. J. Kautsch ${ }^{3}$, A. V. Mosenkov $^{4,5}$, V. P. Reshetnikov ${ }^{5,6}$, \\ N. Ya. Sotnikova ${ }^{5,6}$, N. V. YabloKova ${ }^{5}$, AND R. W. HiLlyeR ${ }^{7}$ \\ ${ }^{1}$ Apache Point Observatory and New Mexico State University, Sunspot, NM, 88349, USA \\ 2 Sternberg Astronomical Institute, Moscow State University, Moscow, Russia \\ ${ }^{3}$ Nova Southeastern University, Fort Lauderdale, FL 33314, USA \\ ${ }^{4}$ Central Astronomical Observatory of RAS, Russia \\ ${ }^{5}$ St. Petersburg State University, Russia \\ ${ }^{6}$ Isaac Newton Institute of Chile, St. Petersburg Branch, St. Petersburg, Russia \\ ${ }^{7}$ Christopher Newport University, Newport News, VA 23606, USA \\ Received 2013 July 20; accepted 2014 March 25; published 2014 April 30
}

\begin{abstract}
We present a catalog of true edge-on disk galaxies automatically selected from the Seventh Data Release of the Sloan Digital Sky Survey (SDSS). A visual inspection of the $g, r$, and $i$ images of about 15,000 galaxies allowed us to split the initial sample of edge-on galaxy candidates into 4768 (31.8\% of the initial sample) genuine edge-on galaxies, $8350(55.7 \%)$ non-edge-on galaxies, and 1865 (12.5\%) edge-on galaxies not suitable for simple automatic analysis because these objects either show signs of interaction and warps, or nearby bright stars project on it. We added more candidate galaxies from RFGC, EFIGI, RC3, and Galaxy Zoo catalogs found in the SDSS footprints. Our final sample consists of 5747 genuine edge-on galaxies. We estimate the structural parameters of the stellar disks (the stellar disk thickness, radial scale length, and central surface brightness) in the galaxies by analyzing photometric profiles in each of the $g, r$, and $i$ images. We also perform simplified three-dimensional modeling of the light distribution in the stellar disks of edge-on galaxies from our sample. Our large sample is intended to be used for studying scaling relations in the stellar disks and bulges and for estimating parameters of the thick disks in different types of galaxies via the image stacking. In this paper, we present the sample selection procedure and general description of the sample.
\end{abstract}

Key words: galaxies: fundamental parameters - galaxies: general - galaxies: photometry - galaxies: spiral galaxies: structure

Online-only material: color figure, machine-readable tables

\section{INTRODUCTION}

Edge-on galaxies provide a unique opportunity for studying the vertical structure of galactic components. Starting from early studies conducted mostly in the optical bands (Kormendy \& Bruzual 1978; Burstein 1979; van der Kruit \& Searle 1981a, 1981b; Kylafis \& Bahcall 1987) using simple photometric profile fitting, the studies of the vertical structure of galactic components evolved toward complex modeling based on radiation transfer methods (Xilouris et al. 1999; Yoachim et al. 2006; Bianchi 2007; Baes et al. 2011; Schechtman-Rook et al. 2012; De Geyter et al. 2013) using multiple UV, optical, and IR data (Popescu et al. 2000; De Geyter et al. 2013). Most of the structural studies employed limited samples of objects using highquality observations. Large surveys conducted during the last decade have made available the benefits of observing large samples of interesting objects, which helps in statistical studies of the vertical structure of galactic disks, bulges, and thick disks (Zibetti et al. 2004; Bergvall et al. 2010). In this paper, we describe our approach to selection of true edge-on galaxies from objects observed by Sloan Digital Sky Survey (SDSS; Aihara et al. 2011). We identified about 6000 genuine edge-on galaxies with inclination angles not more than a few degrees different from perfect edge-on view. Our sample allows statistical studies of the vertical structure parameters of galactic components for the largest sample known to date. We also introduce an online catalog of processed SDSS images and of corresponding structural parameters, which will simplify further studies of edge-on disk galaxies in the optical bands. This paper describes our sample selection procedure and our approach to determination of the stellar disk parameters. The paper is focused mostly on the stellar disk parameters, while bulges will be considered in the next paper.

\section{THE SAMPLE OF EDGE-ON GALAXIES}

\subsection{Selection of Candidates to the Initial Sample}

The initial sample of candidates to edge-on galaxies was automatically selected from the SDSS Seventh Data Release (DR7; Abazajian et al. 2009) using its Catalog Access Server query tools. The selection criteria are discussed in detail by Kautsch et al. (2006a, 2006b) and Kautsch (2009a). This selection was based on the axial ratio, angular diameter, magnitude, and color limits. Flagged galaxies and objects with extreme magnitude errors were not included. The SDSS query was tailored to select relatively bright galaxies with apparent Petrosian magnitudes in the $g$ band less than 20 mag using the Petrosian flux; galaxies with angular major-axis diameters larger than 30 arcsec based upon isoA_g, the isophotal major axis given in SDSS in $g$ band; and "flat" galaxies with an axis ratio $>3$ in the $g$ band, which is defined by the isophotal axes isoA_g divided by isoB_g. The objects are also selected in certain $(-0.5 \leqslant g-r \leqslant 2)$ and $(-0.5 \leqslant r-i \leqslant 2)$ color ranges. The use of the color ranges in reddening-corrected Petrosian magnitudes allows to prevent the inclusion of galaxies with unusual colors caused by active galactic nuclei, instrument flaws, or ghost images. All of these 
selection criteria were applied to the Galaxy table (G.) at the SDSS SkyServer using Structured Query Language (sql). The sql query follows:

$$
\begin{aligned}
& \text { petroMag_g }<20 \\
& \text { G.isoA_g/G.isoB_g }>3 \\
& -0.5<\text { G.dered_g - G.dered_r }<2 \\
& -0.5<\text { G.dered_r }- \text { G.dered_i }<2 \\
& \text { G.isoA_g }>37.8
\end{aligned}
$$

The resulting sample consisted of 18,277 unique objects. A brief visual inspection of the images was done to get rid of false detections. After that, our final sample of the candidates to edge-on galaxies included 14,983 objects.

\subsection{Visual Inspection and the Final Sample of True Edge-on Galaxies}

The photometrically calibrated SDSS frames with selected candidates in the $g, r$, and $i$ bands were taken from the SDSS Data Access Server. By the time we started working with the images, SDSS Data Release 8 (DR8) was issued (Aihara et al. 2011), and the images were downloaded from the DR8. The images were cleaned of foreground stars. The star candidates were identified in the images as objects with FWHM from $1-1.5$ arcsec (typical values during the SDSS imaging campaign; Abazajian et al. (2009)). The stars in the images were replaced by the median values of pixels beyond 3 arcsec (about twice the typical FWHM) from their center. Since the selected images from SDSS are far from really crowded fields, this method of cleaning from stars did not produce strong artifacts. Just a few cases of projected bright stars were caught in the course of the visual inspection, and such galaxies were removed from the consideration. The very central regions of the galaxies were excluded from the cleaning procedure. Having an initial guess about the galactic center coordinates, we fitted ellipses to galactic isophotes at the level of signal-to-noise $\mathrm{S} / \mathrm{N}=2 \mathrm{pixel}^{-1}$ (with the image scale of approximately $0.4 \operatorname{arcsec}_{\text {pixel }}{ }^{-1}$ ). Fitting an ellipse to the outer galactic isophotes allowed us to adjust the position of the galactic center and to determine the size of the "region of interest" that encompasses the whole galaxy (the encompassing ellipse, hereafter). The images were then rotated to align the major axis of the encompassing ellipse with the $x$-axis in the new subframes and then cropped. This allowed us to make a set of images with known geometrical parameters centered on the galaxies, which is necessary for further automatic processing (see Section 3). The images were used for simplification of our visual inspection and also became a part of our catalog (see Section 3.3).

As the next step, all objects were visually classified into groups from the standpoint of further availability for automatic processing. The galaxies with a clearly seen dust layer, or without signs of non-edge-on spiral arms, were classified as true edge-ons. As a result, we selected groups of genuine edge-on galaxies, non edge-ons, objects that needed manual preprocessing (e.g., because a bright star nearby did not allow an automatic algorithm to correctly determine the parameters of the galaxy), and objects that were not suitable for the automatic processing, described below. In other words, we excluded the objects whose galactic midplane could not be aligned along the major axis of a subimage. The latter group includes significantly warped edge-on galaxies and interacting galaxies. This group also includes galaxies with very bright projected stars, whose subtraction would modify a significant part of the galactic image. The initial frames with the objects for the manual processing were then inspected visually, the centers of the galaxies and their orientation parameters were estimated, and properly rotated subframes were made. The galaxies then were classified in the way described above and added to the main sample. The resultant sample was split into 4768 (31.8\%) true edge-on galaxies, $8350(55.7 \%)$ non-edge-ons, and 1865 $(12.5 \%)$ objects that needed a more complex analysis. We do not consider the latter group of the objects in this paper. The nonedge-on galaxies were also excluded from further consideration. This paper is focused on the analysis of the structural parameters of bona fide edge-on, nonwarped, and noninteracting galaxies.

The galaxies were classified into obvious morphological types, from $\mathrm{Sa}$ to Irr using an automatic algorithm, which is described in detail by Kautsch et al. (2006a) and Kautsch (2009a). The major goal of this classification is to assign morphological Hubble types based upon the size of the bulge component since other morphological properties, such as the shape of spiral arms, are obscured at the edge-on view. We use the concentration index and ellipticity of the objects for making this automated classification.

The concentration index (CI) is widely used as a classification criterion, reflecting a measure of the spheroidal component in galaxies (e.g., Pranger at al. 2012; Strateva et al. 2001). We used the CI provided by the SDSS. It is defined as the ratio of the Petrosian radii (petrorad) that contain 90 and 50\% of the Petrosian flux in the $r$ band (Stoughton et al. 2002). The CI in SDSS is measured using circular apertures. This leads to a significant flaw in classification of galaxies with a wide range of viewing angles in surveys that observe all types of galaxies with different inclinations, ranging from face-on to edge-on view. In our work, we focus on purely edge-on disk galaxies. This, in turn, means that all of our objects are affected in the same way, and we do not have to deal with normalizing inclination effects to the CI since our sample is carefully selected to consist of only edge-on galaxies. Kautsch et al. (2006a) found that the CI clearly separates galaxies with an apparent bulge from galaxies without a clear bulge component.

The CI separation values are chosen by visual classification and then have been applied to serve as the limiting values for automated classification. However, pure simple disk galaxies were not detected in a satisfying way so that Kautsch et al. (2006a) introduced a second measure, which allowed to select bulgeless disks without any central spheroidal component. This parameter reflects the ellipticity $(e)$ of the galaxies and was based on luminosity-weighed elliptical isophotes. Also, in this case, Kautsch et al. (2006a) used visual classification to find the best limiting values in order to distinguish the morphological classes according to the eye inspection. The separation of the morphological types is necessarily somewhat arbitrary, and this is in the nature of the classification itself. Kautsch et al. (2006a) chose the limits based on visual classification and applied those to the automatic cataloging, and they also were required to be consistent with similar studies (Karachentsev et al. 1993, 1999). Therefore, our classification should act as an indicator of the dominance of the bulge component translated into the common language of Hubble types. Later (in Figure 8), we will see that the classification reflects the bulge/total ratios derived from the one-dimensional (1D) profile fitting, which confirms the classification method described above.

In Tables 1 and 2, we provide the limiting values of CI and ellipticity, as well as their mean values. Note that the catalog contains a significant number of early-type spirals because we did not limit our selection to flat and bulgeless, 
Table 1

Concentration Index Classification Criteria and Observing Values

\begin{tabular}{lccc}
\hline \hline Type & Lower Limit & Upper Limit & Mean, Error \\
\hline $\mathrm{Sa}$ & 2.70 & $\ldots$ & $3.191 \pm 0.011$ \\
$\mathrm{Sab}, \mathrm{Sb}$ & 2.70 & $\ldots$ & $2.990 \pm 0.005$ \\
$\mathrm{Sc}$ & 2.15 & 2.70 & $2.540 \pm 0.005$ \\
$\mathrm{Scd}$ & 2.15 & 2.70 & $2.498 \pm 0.007$ \\
$\mathrm{Sd}$ & $\ldots$ & 2.70 & $2.403 \pm 0.005$ \\
$\mathrm{Irr}$ & $\ldots$ & 2.15 & $1.894 \pm 0.030$ \\
\hline
\end{tabular}

Table 2

Ellipticity Classification Criteria and Observing Values

\begin{tabular}{lccc}
\hline \hline Type & Lower Limit & Upper Limit & Mean, Error \\
\hline $\mathrm{Sa}$ & $\ldots$ & 0.400 & $0.310 \pm 0.004$ \\
$\mathrm{Sab}, \mathrm{Sb}$ & 0.400 & $\ldots$ & $0.666 \pm 0.003$ \\
$\mathrm{Sc}$ & $\ldots$ & 0.766 & $0.713 \pm 0.003$ \\
$\mathrm{Scd}$ & 0.766 & 0.816 & $0.794 \pm 0.002$ \\
$\mathrm{Sd}$ & 0.816 & $\ldots$ & $0.826 \pm 0.003$ \\
$\mathrm{Irr}$ & $\ldots$ & 0.816 & $0.367 \pm 0.004$ \\
\hline
\end{tabular}

Table 3

Edge-on Galaxies in our Sample by Morphological Types

\begin{tabular}{lc}
\hline \hline Type & Fraction $(\%)$ \\
\hline $\mathrm{Sa}$ & $7.2 \%$ \\
$\mathrm{Sab}, \mathrm{Sb}$ & $32.2 \%$ \\
$\mathrm{Sc}$ & $19.2 \%$ \\
$\mathrm{Scd}$ & $10.8 \%$ \\
$\mathrm{Sd}$ & $28.8 \%$ \\
$\mathrm{Irr}$ & $1.8 \%$ \\
\hline
\end{tabular}

late-type spirals due to our choice of selection criteria, as discussed in Section 2.1. A summary of the fraction of different morphological types in the sample is shown in Table 3.

Since the initial automatic selection of the galaxy candidates did not include a significant number of the largest edgeon galaxies, we incorporated more edge-on candidates by supplying objects from the Revised Flat Galaxy Catalog (RFGC hereafter; Karachentsev et al. 1999), RC3 (de Vaucouleurs et al. 1991), EFIGI (Baillard et al. 2011), and Galaxy Zoo (Lintott et al. 2011) that were found in the SDSS footprints. The reason for missing extended objects in the SDSS fields lies in the difficulty of assigning correct borders to large and extended objects with multiple luminosity centers, e.g., H II regions. This so-called shredding results in detecting those missed galaxies as two or more entries by SDSS's image processing pipeline.

Only the galaxies with major axes greater than 30 arcsec (according to the HyperLeda database; Paturel et al. 2003) were added. All new edge-on candidates were processed in the way described above and then visually inspected. The final sample of unique true edge-on galaxies consists of 5747 objects. Figure 1 shows that the galaxies in our sample are more or less uniformly allocated in the SDSS imaging survey area.

We checked the completeness of our catalog using the procedures by Thuan \& Seitzer (1979). Their $V / V_{m}$ value was calculated for the whole sample, and we found that our genuine edge-on subsample is $95 \%$ complete for all galaxies with major axes size larger than 28 arcsec. Figure 2 (top curve) shows the histogram of the distribution of the major axes. Our morphological classification allows for splitting the histogram by types, which is shown in Figure 2. Galaxies of different morphological types observed edge-on may be affected by dust

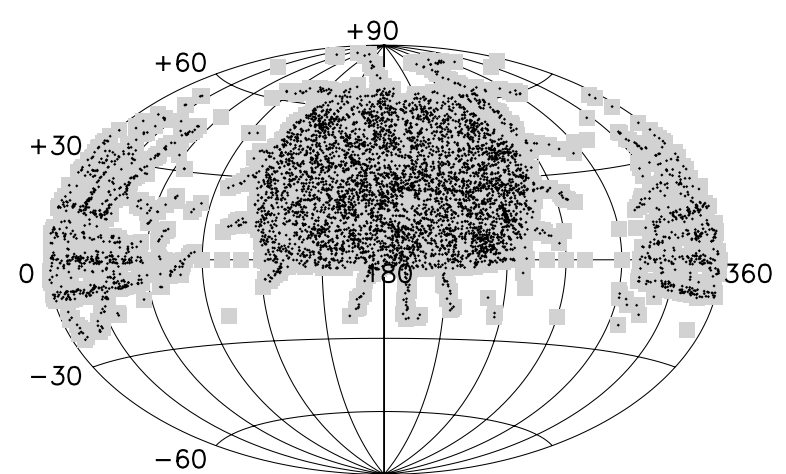

Figure 1. Distribution of genuine edge-on galaxies in the sky shows that they cover R.A.-Decl. space essentially the same way as all SDSS objects do, according to Aihara et al. (2011) and sdss3.org. The grey shaded area designates the SDSS imaging footprints.

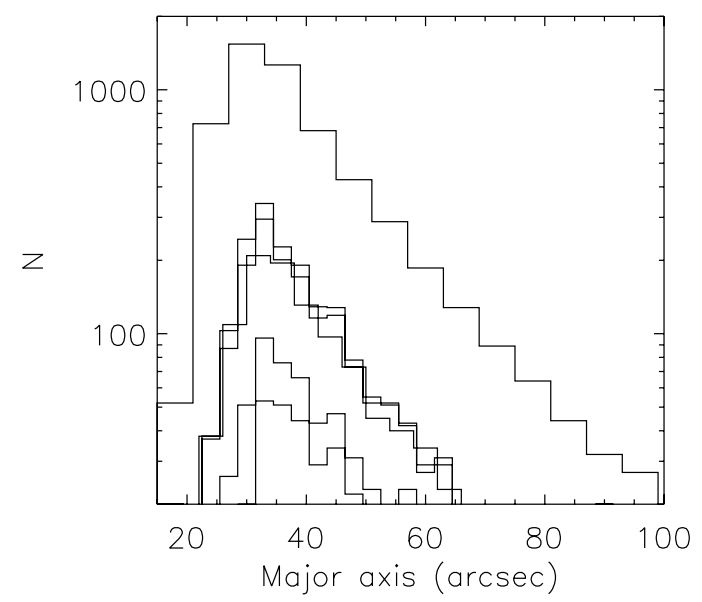

Figure 2. Distribution of the galactic major axis size that was estimated from the SDSS images at $\mathrm{S} / \mathrm{N}=2$; see the text. The distribution (top curve) suggests that our sample is complete for the galaxies with major axes greater than 28 arcsec. Different morphological types are shown below the general sample and correspond to $\mathrm{Sb}, \mathrm{Sd}, \mathrm{Sc}, \mathrm{Scd}$, and $\mathrm{Sa}$ from top to bottom, respectively.

in a different way but we see that different types follow the general distribution. We also calculated the Thuan \& Seitzer's $V / V_{m}$ as a function of the morphological types and found that the $\mathrm{Sa}, \mathrm{Sb}, \mathrm{Sc}, \mathrm{Scd}$, and Sd types are $95 \%$ complete for the galaxies with major axes greater than $33,28,26,25$, and 27 arcsec, respectively. The Irr subsample has low statistics which not enough for certain $V / V_{m}$ calculations. Thus, we conclude that the completeness is not a strong function of morphological type.

We performed a test of how well we select true edge-on galaxies among objects with arbitrary inclination. For this purpose, we submitted a DR7 Catalog Archive Server (CAS) query with the same sample selection criteria except the axis ratio limit. As a result, the output comprises 161,571 objects. The inclination angle, $i$, was coarsely estimated from the minorto-major axes ratio $(b / a)$ using equation $\cos ^{2}(i)=\left((b / a)^{2}-\right.$ $\left.q^{2}\right) /\left(1-q^{2}\right)$ (Hubble 1926), where we assume that the intrinsic axial ratio in the galactic disks is the same for all objects and is equal to $q=0.13$ (Giovanelli et al. 1994), and the major and minor axes, $a$ and $b$, are the $r$ band sizes from the DR7 CAS tables. Figure 3 shows the distribution of the formally estimated $\cos (i)$, which has to be flat in the case of equal probability of the galactic inclinations in space. Figure 3 demonstrates that the variety of edge-on galaxies cannot be described by a single universal value of the internal flatness, $q$. It is seen that due 


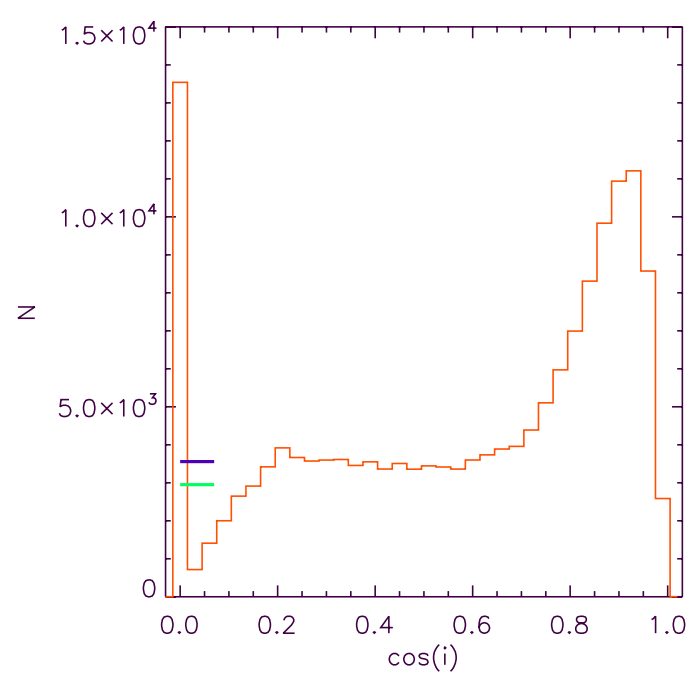

Figure 3. Cosine of the inclination angle in the galaxies selected from SDSS DR7 formally estimated from the major-to-minor axes ratio (see the text) is shown as the solid line histogram. The lower short bar at the highest inclinations designates the number of our true edge-on galaxies estimated from the objects selected with the SDSS CAS query. The upper short bar designates the total number of real edge-on galaxies in our final sample.

(A color version of this figure is available in the online journal.)

to contamination from non-edge-on galaxies with inclination between $80^{\circ}$ and $86^{\circ}$, the inclination distribution strongly peaks at $90^{\circ}$. At the same time, the object migration to the peak creates the dip between $80^{\circ}$ and $86^{\circ}$.

Our visual inspection allowed us to select a statistically reasonable sample of true edge-on galaxies: assuming that we classify a galaxy as a genuine edge-on if its inclination is over $86^{\circ}$ (de Grijs et al. 1997; Bizyaev \& Kajsin 2004), the corresponding number is calculated from the size of our true edge-on sample and is designated by the lower short bar at $86^{\circ}-90^{\circ}$ in Figure 3 . The upper short bar shows the number of the galaxies in our full resulting sample (i.e., the sample with further additions beyond the automatic CAS SDSS selection). The shape of the distribution is the same as in other studies based on SDSS samples, e.g., as in Masters et al. (2010). Note that the peak at the left side of the distribution (where $\cos (i)$ is close to 1 ) shows a growing contribution of elliptical galaxies to the general sample. This does not affect the high inclination end of the diagram. Note that Figure 3 is an illustration that shows selection effects only in the case when an oversimplified approach is applied to the inclination determination. The procedure of our visual inspection provides a more robust way of selection of the edge-on galaxies.

\section{THE STRUCTURAL PARAMETERS FROM THE 1D ANALYSIS OF PHOTOMETRIC PROFILES}

We performed the analysis of 1D photometric profiles using the same technique as described by Bizyaev \& Mitronova (2002, 2009). The volume brightness in the stellar disks is assumed to change as follows in the radial $r$ and vertical $z$ directions:

$$
\rho_{L}(r, z)=\rho_{L_{0}} \exp (-r / h) \operatorname{sech}^{2}\left(z / z_{0}\right),
$$

where $h$ is the scale length and $z_{0}$ is the scale height of the disk. The central face-on disk luminosity, $I_{0}=\int_{-\infty}^{\infty} \rho(0, z) d z$, corresponds to the central surface brightness, $S_{0}$. The model photometric profiles are obtained by the integration of Equation (1) along the line of sight and by the convolution with the instrumental profile. We assume that the point-spread function (PSF) is equal to 1.4 arcsec for all considered images, given the survey photometry campaign description (Aihara et al. 2011). The central regions of the galaxies (one-fourth of the semimajor axis of the encompassing ellipse) in which bulges can be seen are excluded from the stellar disk parameters evaluation in all galaxies. The best-fit scales and surface brightness are estimated from the radial or vertical profiles that were drawn through each pixel row and column within the encompassing ellipses in the cleaned and rotated subframes. Although we did not take the inclination angle into account in this 1D approach, our visual inspection should select the galaxies with a small deviation from the $90^{\circ}$ inclination. As it has been shown by Barteldrees \& Dettmar (1994), de Grijs (1998), and Kregel et al. (2002), the small deviation from the perfect edge-on view does not significantly affect the structural parameters.

We also evaluate and find the structural parameters separately in two halves of the galactic images (as seen above and below the galactic midplane). An axisymmetric and dustless galaxy, even observed at a small angle with respect to the perfect edge-on view, will have the same brightness for the parts seen above and below the galactic midplane. In the presence of dust extinction, the galaxy's halves above and below the midplane look different for observers, see, e.g., Xilouris et al. (1999) and Bianchi (2007). To mitigate effects of the dust, we consider the scales and surface brightness only for the brightest half of each galaxy. Note that because of our selection procedure, there is no big difference between the parameters determined from the brightest half only and from the entire galaxy. The output structural parameters reported by us are the median values of all considered photometric profiles.

Once the structural parameter difference in the near-IR (NIR) photometric bands is affected by dust attenuation (Bizyaev \& Mitronova 2009), the difference in the optical bands is also affected by the gradients of the stellar population. This makes us consider the structural parameters in the $g, r$, and $i$ bands separately from each other.

We also coarsely estimate the contribution of the bulge to the luminosity of the galaxies. Using the estimated $S_{0}, h$, and $z_{0}$, we create images of edge-on disks and subtract them from the images of the galaxies. The bulge-to-total luminosity ratio is found as the luminosity of the residual image integrated over the region within $1.0 \mathrm{~h}$ from the center normalized by the total luminosity of the galaxy integrated within the encompassing ellipse. The structural parameters estimated from the 1D profile analysis for our sample of genuine edge-on galaxies are shown in Table 4.

Comparison with the RFGC shows that our catalog has 917 RFGC objects. The vertical and radial sizes of the encompassing ellipses are well-correlated with the size of the galaxies visually estimated in RFGC: our $r$-band semimajor axis size is $0.95 \pm$ 0.13 of the red semimajor axis for the common galaxies, and our semiminor size is $1.27 \pm 0.21$ of the semiminor axis for them. We matched our catalog to the sample by Bizyaev \& Mitronova (2009) and found 53 objects in common. Our $h$ and $z_{0}$ well correlate with the same structural parameters determined by Bizyaev \& Mitronova (2009) for those 53 galaxies; on average, our radial scale length in the $i$ is $1.11 \pm 0.43$ of that in the $J$ band, and the vertical scale height is $1.20 \pm 0.15$ of the $J$-band scale from Bizyaev \& Mitronova (2009). For the 20 common galaxies with Mosenkov et al. (2010), the agreement is not as strong: the ratio of our scale length to the published one is $1.11 \pm 0.25$, and the ratio of the scale heights is $1.42 \pm 0.41$ for the $J$-band images. 
Table 4

The Structural Parameters of True Edge-on Galaxies From The 1D Analysis

\begin{tabular}{|c|c|c|c|c|c|c|c|c|c|c|c|c|}
\hline $\begin{array}{l}\text { Name } \\
B / T\end{array}$ & $\begin{array}{l}\text { Band } \\
\text { Type }\end{array}$ & R.A. (J2000) & Decl. (J2000) & P.A. & $h$ & $d h$ & $z_{0}$ & $d z_{0}$ & $S_{0}$ & $d S_{0}$ & $\operatorname{grad}\left(z_{0}\right)$ & mag \\
\hline $\begin{array}{l}\text { EON_0.183_7.090 } \\
0.14\end{array}$ & $\begin{array}{c}g \\
\text { SB }\end{array}$ & 0.182590 & 7.090147 & 157.78 & 7.31 & 0.61 & 1.21 & 0.19 & 23.75 & 0.26 & -0.194 & 17.69 \\
\hline $\begin{array}{l}\text { EON_0.183_7.090 } \\
0.18\end{array}$ & $\begin{array}{c}i \\
\mathrm{SB}\end{array}$ & 0.182590 & 7.090147 & 157.78 & 5.56 & 1.14 & 1.30 & 0.20 & 22.82 & 0.50 & -0.104 & 16.49 \\
\hline $\begin{array}{l}\text { EON_0.183_7.090 } \\
0.09\end{array}$ & $\begin{array}{c}r \\
\mathrm{SB}\end{array}$ & 0.182590 & 7.090147 & 157.78 & 7.61 & 2.56 & 1.31 & 0.18 & 23.51 & 1.04 & -0.230 & 16.88 \\
\hline $\begin{array}{l}\text { EON_0.187_33.757 } \\
0.08\end{array}$ & $\begin{array}{c}g \\
\mathrm{SB}\end{array}$ & 0.186788 & 33.756809 & 156.87 & 2.55 & 0.17 & 0.91 & 0.09 & 21.80 & 0.15 & 0.012 & 18.23 \\
\hline $\begin{array}{l}\text { EON_0.187_33.757 } \\
0.15\end{array}$ & $\begin{array}{c}i \\
\mathrm{SB}\end{array}$ & 0.186788 & 33.756809 & 156.87 & 3.16 & 0.06 & 0.85 & 0.09 & 21.30 & 0.28 & -0.048 & 16.90 \\
\hline $\begin{array}{l}\text { EON_0.187_33.757 } \\
0.11\end{array}$ & $\begin{array}{c}r \\
\mathrm{SB}\end{array}$ & 0.186788 & 33.756809 & 156.87 & 3.59 & 0.53 & 0.88 & 0.10 & 22.06 & 0.19 & 0.061 & 17.36 \\
\hline$\cdots$ & & & & & & & & & & & & \\
\hline
\end{tabular}

Notes. Parameters of the galaxies in the table: EGIS name, SDSS band, R.A. (J2000) in decimal degrees, Decl. (J2000) in decimal degrees, position angle, scale length in arcsec and its uncertainty, scale height in arcsec and its uncertainty, face-on central surface brightness and its uncertainty, vertical gradient of the scale height normalized by the scale ratio $\left(d z_{0} / d r *\left(h / z_{0}\right)\right)$, total uncorrected magnitude of the galaxy estimated by integration within the encompassing ellipse, the bulge-to-total ratio, morphological type of galaxies, heliocentric radial velocity in $\mathrm{km} \mathrm{s}^{-1}$ (from LEDA; -1 is inserted if the value is unknown), and an alternative name.

(This table is available in its entirety in a machine-readable form in the online journal. A portion is shown here for guidance regarding its form and content.)

The sample by Yoachim et al. (2006) has 18 common galaxies with our catalog. Our structural parameters were determined using the same functional form for the radial and vertical profiles, and the results are in reasonable agreement: our radial scale length is $1.23 \pm 0.30$, and our vertical scale height is $0.99 \pm 0.13$ of those from the $R$-band estimates by Yoachim et al. (2006).

The parameters for one common thick early type disk galaxy with Pohlen et al. (2004) are also in good agreement (1.03 and 1.07 for the radial and vertical scales ratio, respectively, between our $g$ and their $V$ images). Our scale length is $1.07 \pm 0.36$ of that found for the large galaxies by Bianchi (2007). On the other hand, our scale height is much thicker than that reported by Bianchi (2007).

\subsection{The Structural Parameters}

The histogram of the distribution of the radial-to-vertical scale ratios $h / z_{0}$ is shown in Figure 4. The distribution has a prominent peak at $h / z_{0} \approx 2.5$ in the $r$ band. The median values over the whole sample are 3.6, 3.4, and 3.3 for the $g, r$, and $i$ bands, respectively. The radial-to-vertical scale ratios in Figure 4 are somewhat lower than those for the typical edge-on galaxies estimated from the NIR (Bizyaev \& Mitronova 2002, 2009). For the sample of 153 galaxies composed by Bizyaev \& Mitronova (2002), the median ratio of $h / z_{0}$ is about 4.8. This is expected because we do not constrain the bulge contribution and consider a wide range of disk galaxies (see Figure 6), whereas Bizyaev \& Mitronova $(2002,2009)$ focused on bulgeless galaxies. The scale ratios in our sample are consistent with the NIR data presented by Mosenkov et al. (2010). Mosenkov et al. (2010) constructed a sample of 175 edge-on galaxies both of early- and late-types and found the median ratio $h / z_{0}$ to be about 3.5 in the $J$ band and 3.9 for the $H$ and $K_{\mathrm{s}}$ bands.

The distribution of the face-on central surface brightness, $S_{0}$, in the gri bands is shown in Figure 5. The surface brightness was corrected for reddening in our Galaxy using the extinction maps by Schlegel et al. (1998). It is seen that the central surface brightness values, $S_{0}$, span five magnitudes in each

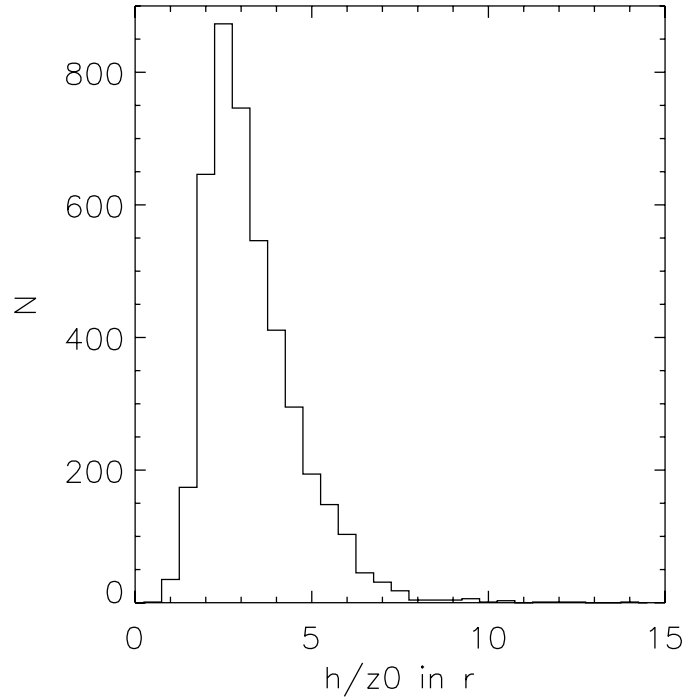

Figure 4. Distribution of the inverse stellar disk thickness $h / z_{0}$ estimated from the $r$-band images for all galaxies whose $z_{0}$ is greater than 3 pixels.

band. Apparently, our central surface brightness is biased toward the dimmer values with respect to those for arbitrary inclined galaxies, which is a manifestation of the dust extinction within the galaxies. It suggests that a more complex modeling will help in better recovery of the stellar disk central brightness from the data of optical photometry.

Although the bulge contribution is a parameter affected by dust and projection effects in edge-on galaxies, the bulge-to-disk luminosity ratio is helpful in morphological classification, since the spiral arms cannot be observed in edge-on stellar disks. We show the $B / T$ ratio for the galaxies in our sample in Figure 6 with a warning of using this value with caution for direct comparison with arbitrary inclined galaxies. A more honest $B / T$ ratio can be recovered from three-dimensional (3D) modeling by including the central area of galactic images into the analysis. The inverse scale ratio $h / z_{0}$ reveals some dependence of the bulge-to-total 


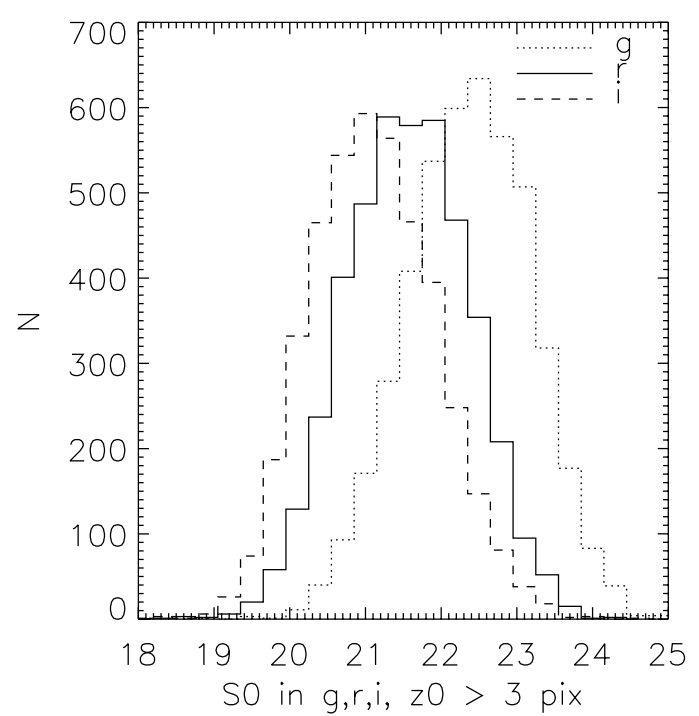

Figure 5. Distribution of the stellar disk's central face-on surface brightness. The dotted, solid, and dashed curves designate the distributions in the $g, r$, and $i$ bands, respectively. The surface brightness was corrected for the foreground reddening in our Galaxy.

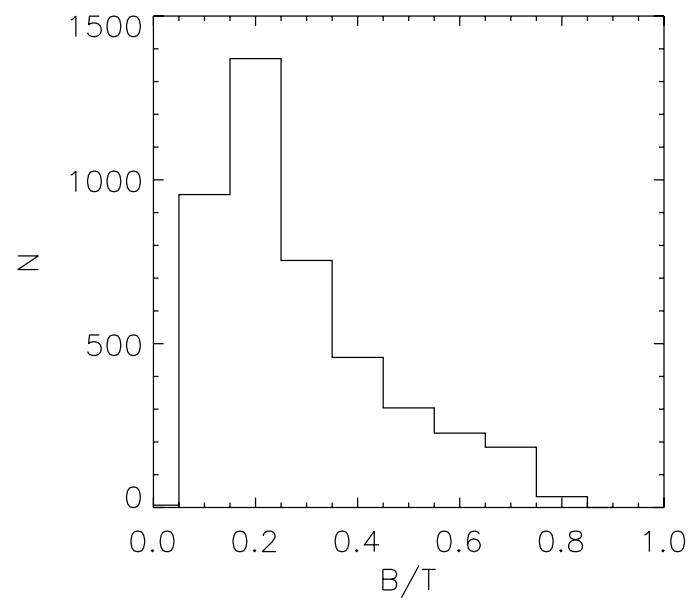

Figure 6. Distribution of the bulge-to-total luminosity ratio, $B / T$. The ratio is estimated from the $r$-band images of all galaxies whose $z_{0}$ is greater than 3 pixels.

ratio, whereas our 3D analysis shows that the trend is much less significant.

The disk central surface brightness determined for the brighter and dimmer halves of the galaxies allows us to introduce the "brightness asymmetry" parameter, estimated as the difference between the brighter and the dimmer values of the central surface brightness. Although it is a function of the galactic inclination in the case of an individual galaxy, in a combination with variable disk thickness, bulge strength, and clumpy nature of the dust layer, it is not a direct measure of the inclination in a sample of edge-on galaxies. Nevertheless, the asymmetry gives a possibility to test if our selection procedure is biased and gives preference to certain values of the asymmetry. We ran the $V / V_{m}$ test as in Section 2.2 for several groups of galaxies with similar asymmetry Asy in the $r$ band: $0<A s y<0.14 \mathrm{mag}$, $0.14 \mathrm{mag}<A s y<0.30 \mathrm{mag}, 0.30 \mathrm{mag}<A s y<0.49 \mathrm{mag}$, $0.49 \mathrm{mag}<A s y<0.75 \mathrm{mag}$, and Asy $>0.75 \mathrm{mag}$. Each of the five groups contains an approximately equal number of the members, which is one-fifth of the whole sample. The $95 \%$ completeness level is achieved for major axes greater than $30,29,29,28$, and 27 arcsec, respectively. It indicates that

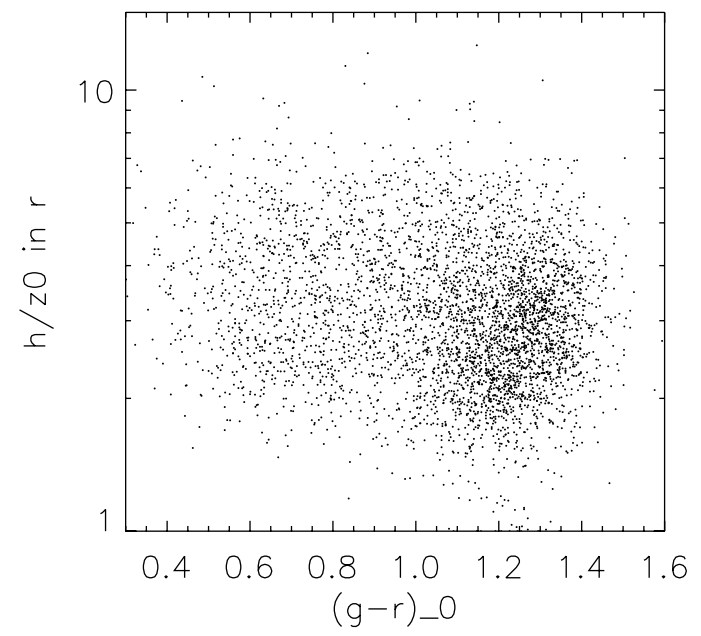

Figure 7. Inverse stellar disk thickness $h / z_{0}$ (in the $r$ band) vs. the corrected color $(g-r)$ for all galaxies in our sample. The colors are corrected for reddening in our Galaxy.

the galaxies with different asymmetries have mostly the same completeness as a whole sample of galaxies. We checked if the asymmetry is a function of disk thickness, central surface brightness, or distance (for those galaxies with available radial velocities) and did not find any such trends. Thus, we select the objects uniformly from the standpoint of their bright-dim halves asymmetry.

We also sorted out the galaxies by their inverse disk scale ratio $h / z_{0}$ in the $r$ band and considered completeness using the $V / V_{m}$ test. The $h / z_{0}$ ranges of $(0.0,2.4),(2.4,3.1),(3.1,3.9)$, $(3.9,5.1)$, and over 5.1 made the groups of mostly equal size, and their $95 \%$ completeness level starts at the major axis size of 29, 28, 29, 30, and 28 arcsec, respectively. This shows that our sample is not biased over the internal disk thickness.

The galactic colors were estimated using equal areas within the encompassing ellipses. The colors were corrected for the Milky Way reddening using the maps from Schlegel et al. (1998) but they were not corrected for the internal extinction in the galaxies. We do not observe significant trends in the disk thickness with the overall galaxy color (Figure 7), although red objects tend to possess thicker disks, similar to that reported by Kautsch (2009b).

The colors, as well as the bulge-to-disk luminosity ratio, correlate well with the estimated morphological type in our sample (see Figure 8).

More details on the statistical properties of the derived structural parameters are presented in Table 4.

\subsection{The Scale Height Gradient}

Since we analyzed the vertical photometric profiles independently of each other, we can estimate how the vertical scale height changes with the distance to the center in terms of the scale height radial gradient. The gradient is calculated from individual vertical photometric profiles in the range from 1 to 3 radial scale lengths. Figure 9 (lower panel) shows the distribution of the scale height gradient for the whole sample. The gradient was normalized and is shown as $\left(d z_{0} / d r\right) /\left(h / z_{0}\right)$. The distribution peaks close to zero, at small positive values of the gradient: the mean value is 0.063 , the median is 0.064 , and the mode is 0.067 . The fraction of galaxies with strong positive and negative radial gradients of the $z_{0}( \pm 0.2$ from the median value) is $10.8 \%$. It is interesting to note that large positive gradients 

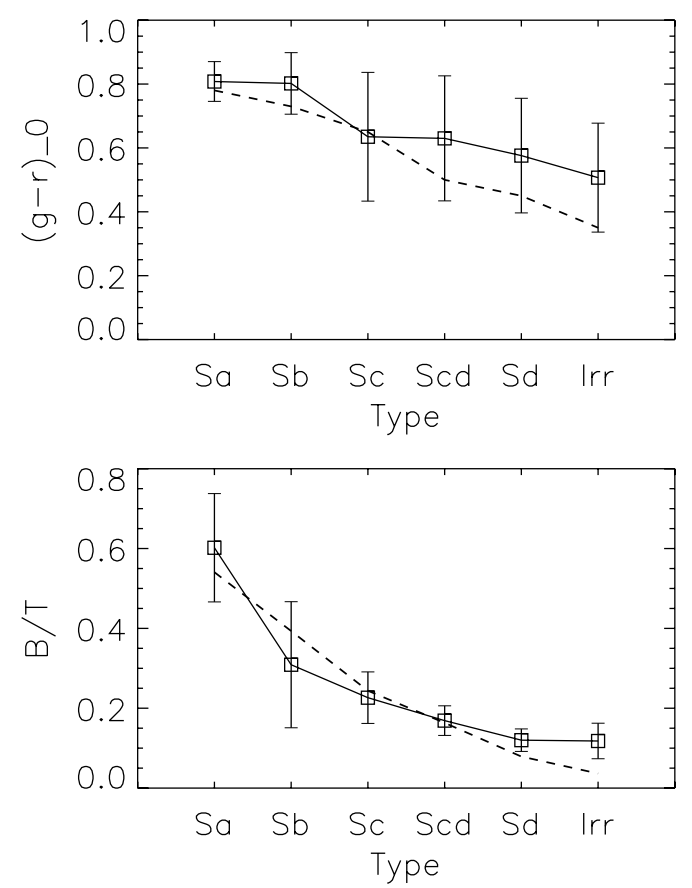

Figure 8. Mean values and rms scatter of the $(g-r)$ color (top) and of the bulge-to-disk luminosity ratio (bottom) for different morphological types in our sample. The dashed curve in the top panel shows the color morphological type dependence for the same isolated galaxies with arbitrary inclination from Fernández Lorenzo et al. (2012). The dashed curve in the bottom panel denotes the bulge-to-total luminosity distribution from the EFIGI sample (Baillard et al. 2011).
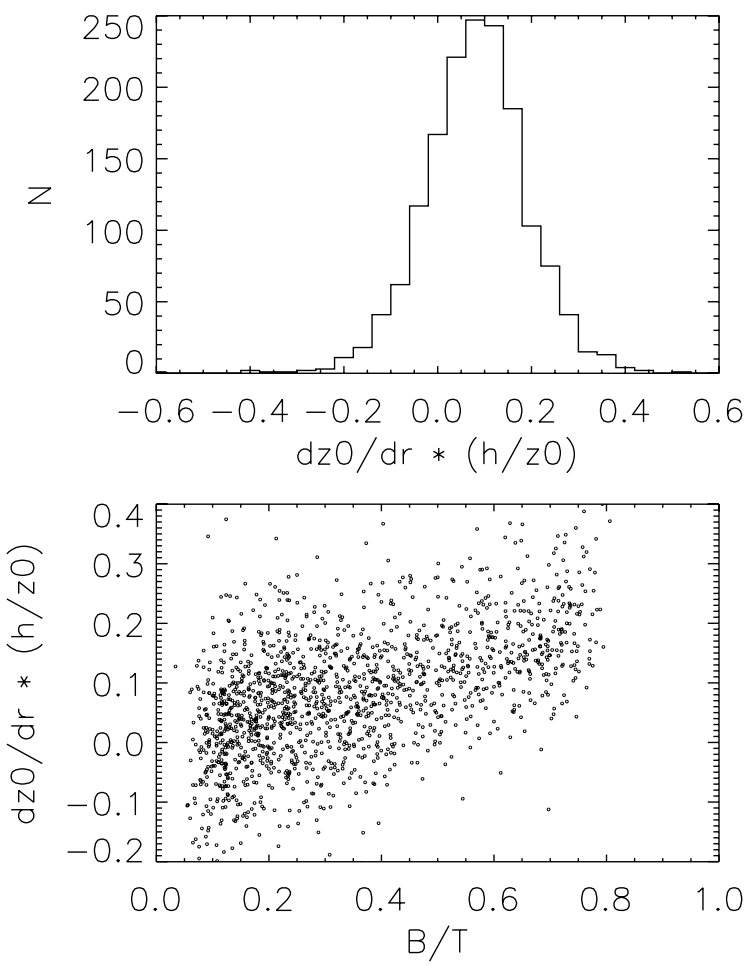

Figure 9. Top: the radial gradient of the stellar disk thickness $z_{0}$ estimated from the $r$-band images. The gradient $d z_{0} / d r$ is normalized by the disk thickness $z_{0} / h$. Bottom: the normalized radial gradient of the scale height vs. the bulgeto-disk ratio.

of the scale height are observed mostly in the galaxies with significant bulges, whereas bulgeless galaxies have disks without the radial gradients of the vertical scale, on average. This trend suggests that large bulges affect our method of the gra-
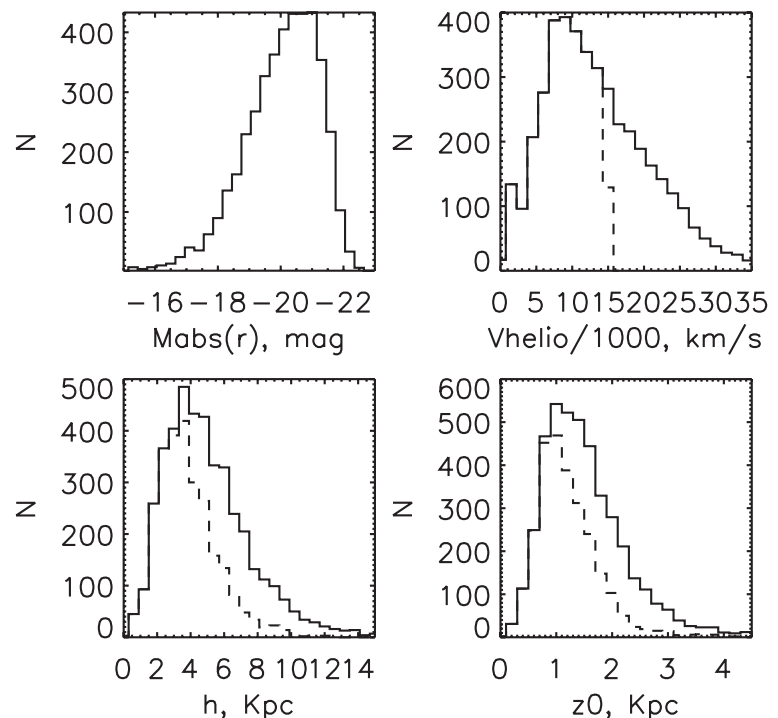

Figure 10. Distribution of the absolute magnitude in the $r$ band, radial velocity, and the radial $(h)$ and vertical $\left(z_{0}\right)$ scales expressed in kpc. The absolute magnitude is corrected for reddening in the Milky Way. The $h$ and $z_{0}$ distributions for the whole sample are designated by the solid line, whereas the dashed line marks those distributions for relatively nearby galaxies with heliocentric velocity $V_{\text {helio }}<15,000 \mathrm{~km} \mathrm{~s}^{-1}$.

dient determination, and the gradients inferred for the galaxies with $B / T>0.4$ are biased. For the galaxies with $B / T<0.4$, the normalized radial gradient in the $r$ band is 0.045 on average. Given the bulge contamination and dust effects, we should warn the readers about the limitations of the gradient determination, especially in the case of the smallest galaxies in our sample. The vertical scale gradients can be addressed with additions to a 3D modeling approach, which we will introduce to the modeling in the next paper.

\subsection{The Catalog of True Edge-on Galaxies}

We present our sample of edge-on galaxies as an online catalog EGIS (Edge-on Galaxies In SDSS), publicly available at http://users.apo.nmsu.edu/ dmbiz/EGIS/. The catalog's core table is our Table 4, which contains the structural parameters for each image in the $g, r$, and $i$ bands. The catalog also contains cleaned images used for our analysis of structure, as well as raw (not cleaned) images. All images are trimmed to have the galactic center at the center of the frame and are rotated to place the major axis parallel to the image rows. Note that all initial images for the analysis are taken from the SDSS, so all SDSS data usage policies are applied to our catalog.

Cross-matching over the HyperLeda database allows us to find radial velocities for about three-fourths of our sample. The distribution of the physical properties (the absolute magnitude in the $r$ band, the radial velocity, and the radial and vertical scales in the physical units) is shown in Figure 10.

The future implications of the catalog include co-adding images in order to study properties of the thick disks statistically, evaluation of the bulge properties, and study of scaling relations based on the large sample of similar objects.

\section{STRUCTURAL PARAMETERS FROM 3D MULTICOMPONENT MODELING}

Realistic modeling of edge-on galaxies for the structural parameters determination must include the dust extinction. As modeling of some large galaxies shows (Xilouris et al. 1999; 
Table 5

The Structural Parameters Derived from the 1D Profile Analysis

\begin{tabular}{lccr}
\hline \hline Parameter & \multicolumn{1}{c}{$g$} & \multicolumn{1}{c}{$r$} & \multicolumn{1}{c}{$i$} \\
\hline$h / z_{0}$ & $3.57 \pm 1.61$ & $3.31 \pm 1.31$ & $3.17 \pm 1.21$ \\
$S_{0}$ & $22.37 \pm 0.81$ & $21.56 \pm 0.81$ & $21.10 \pm 0.84$ \\
$B / T$ & $0.24 \pm 0.16$ & $0.29 \pm 0.17$ & $0.31 \pm 0.17$ \\
$h(\operatorname{arcsec})^{\mathrm{a}}$ & $5.9 \pm 2.3$ & $5.5 \pm 2.0$ & $5.3 \pm 1.9$ \\
$z_{0}(\operatorname{arcsec})$ & $1.8 \pm 0.6$ & $1.8 \pm 0.5$ & $1.8 \pm 0.5$ \\
\hline
\end{tabular}

Notes. Mean values and uncertainties of the reverse thickness $h / z_{0}$, central surface brightness, and bulge-to-total ratio estimated for different SDSS bands. ${ }^{a}$ Median value for the scale length and scale heights in arcsec are given with their uncertainty calculated as $1.48 \times$ MAD (median absolute deviation).

Popescu et al. 2000; Yoachim et al. 2006; Bianchi 2007), the dust in the galaxies can be successfully approximated as an embedded disk with uniform extinction and scattering. As Bianchi (2007) noticed, neglecting the dust scattering and taking into account only dust absorption does not introduce significant errors to the resulting structural parameters. We simplify calculations and neglect the dust scattering in the modeling described below and assume that the dust extinction volume density can be expressed as

$$
\kappa_{\mathrm{ext}, \lambda}(r, z)=\frac{\tau_{0, \lambda}}{2 z_{\mathrm{d}}} \exp \left(-r / h_{\mathrm{d}}\right) \exp \left(-|z| / z_{\mathrm{d}}\right) .
$$

Here, $\tau_{0, \lambda}$ is the face-on optical depth of the dust disk at the center, and $h_{\mathrm{d}}$ and $z_{\mathrm{d}}$ are the radial and vertical scales of the dust disk. The distribution of the luminosity density in our model stellar disks follows Equation (1). We also add a stellar bulge to the modeling since we did not limit our consideration by bulgeless galaxies, and many of our galaxies have noticeable bulge (according to Figure 6 and Table 5). We assume the Hubble volume luminosity density distribution for the bulge (Xilouris et al. 1998), which can be written

$$
\rho_{\mathrm{b}}(r, z)=\rho_{0 \mathrm{~b}}\left(1-B^{2}\right)^{-3 / 2},
$$

where $B=\left(\sqrt{r^{2}+z^{2}(b / a)^{2}} / R_{\mathrm{e}}\right), \rho_{0 \mathrm{~b}}$ is the central luminosity density in the bulge, and $R_{\mathrm{e}}$ is the bulge effective radius.

After adding the co-planar embedded extinction disk and tilting the system by close to an edge-on inclination angle, we calculate the final brightness distribution via numerical integration of the luminosity volume density along the line of sight. The 2-D images were convolved with a Gaussian PSF, the same as we used in Section 3.

Attempts of modeling the stellar disk using SDSS images with an unconstrained set of parameters was performed by Bizyaev (2007) using chi-square minimization of the difference between the real and model galactic images. As modeling of small samples of well spatially resolved galaxies shows (Xilouris et al. 1999; Matthews et al. 1999; Yoachim et al. 2006; Bianchi 2007), the dust disk described by Equation (2) has two times smaller scale height than that in the stellar disks. To simplify calculations, we assume that $z_{\mathrm{d}}=z_{0} / 2$ in further 3D modeling.

Our model has 14 free parameters: the $X-Y$ position of the center in the sky plane, the position angle (P.A.) of the major axis, the inclination of the galactic plane, central surface brightness and the scales of the stellar disk, central face-on extinction and the scales of the dust disk, and central surface brightness, axes ratio, and effective radius of the bulge.

To ensure that we can reliably recover the galactic component parameters, we performed Monte Carlo simulations and created a set of synthetic images of edge-on galaxies. Using
Equations (1), (2), and (3), we made a large set of synthetic images and projected them to the sky plane by adding some inclination different from the perfect edge-on view and a small P.A. tilt. A random noise was added to the images in order to degrade the image quality and to make them have $\mathrm{S} / \mathrm{N}$ comparable to the observing data. The synthetic images were then evaluated, and recovered parameters were compared to the input parameters (see Figure 11).

As the modeling in Figure 11 shows, not all parameters are recovered equally well: the structural parameters of the stellar disks are the most reliable ones. The dust disk parameters are not reliably estimated. The inclination of the galactic plane is the hardest parameter to estimate even from smooth and nonclumpy synthetic images.

We ran the same simulations with variable pixel size of the synthetic images to understand how small the galaxies could be in order to be suitable for the analysis. Figure 12 demonstrates the stellar disk scales and the central surface brightness (in arbitrary linear units) estimated from synthetic images. We created 21 images for a set of scale height, and estimated resulting structural parameters from our 1D and 3D analysis. As Figure 12 suggests, reliability of both approaches is bad when the scale height is comparable with the pixel resolution in the images. The 1D analysis overestimates the disk thickness by $15 \%$, given sufficient pixel resolution, which is due to the combination of non-perfect edge-on galactic inclination and effects of the dust layer. The difference in the scales determined using these two approaches can be seen in the real data in Section 4.1.

The distribution of the radial-to-vertical scale ratio is shown in Figure 13 for relatively large galaxies (with $z_{0}>1.2$ arcsec). The stellar disks look thinner from the 3D analysis results in comparison with Figure 4. The disk thickness estimated from different bands with the 3D analysis looks similar. The median inverse disk thickness is 5.7, 5.8, and 5.6 in the $g, r$, and $i$ bands, respectively.

Since morphological dependence of the stellar disk thickness has been reported from smaller sample studies (de Grijs 1998; Schwarzkopf \& Dettmar 2000), we check how the average disk thickness is correlated with our preliminary morphological classification. Figures 14 and 15 compare the median inverse disk thickness estimated for different morphological types. Figure 14 uses the results of our 1D analysis, whereas Figure 15 is based on the results from the $3 \mathrm{D}$ analysis. It can be seen that Figure 14 is in very good agreement with Figure 6 from de Grijs (1998) (shown with filled circles) and close to the results by Schwarzkopf \& Dettmar (2000). Figure 15 reveals thinner stellar disks in the late-type spiral galaxies. It also suggests that the galactic extinction contributes to the formation of the trend seen in Figure 14.

The stellar disk structural parameters are estimated for the same galaxies by the 1D and 3D approaches independently from each other, so it is worth comparing the resultant parameters. Figure 16 shows the comparison for the stellar disk scale length $h$, scale height $z_{0}$, and face-on central surface brightness $S_{0}$. The vertical scale height in the same images is smaller in the $3 \mathrm{D}$ case, which suggests that taking the dust into consideration improves the analysis. In combination with the correction of the central surface brightness for the internal extinction, our 3D approach produces significantly brighter stellar disks in the comparison with the $1 \mathrm{D}$ analysis.

Both our 1D and 3D approaches to the modeling are affected by limited spatial resolution of SDSS images, which is severe 

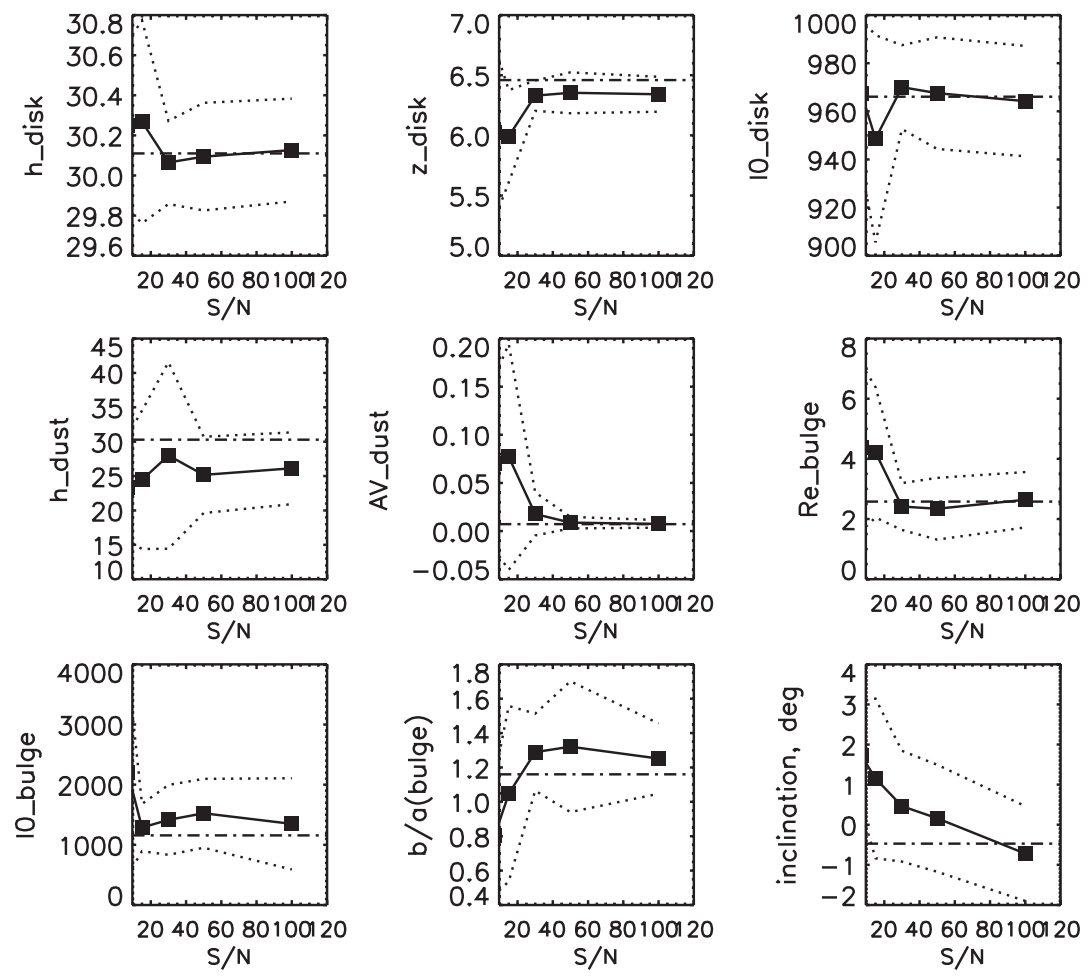

Figure 11. Model parameters recovered from our synthetic images. The solid curve with symbols shows the averaged output parameters, The dashed line designates the input value. The dotted lines designate the $1 \sigma$ uncertainty of the parameters estimated from 30 simulations for each $\mathrm{S} / \mathrm{N}$ ratio.
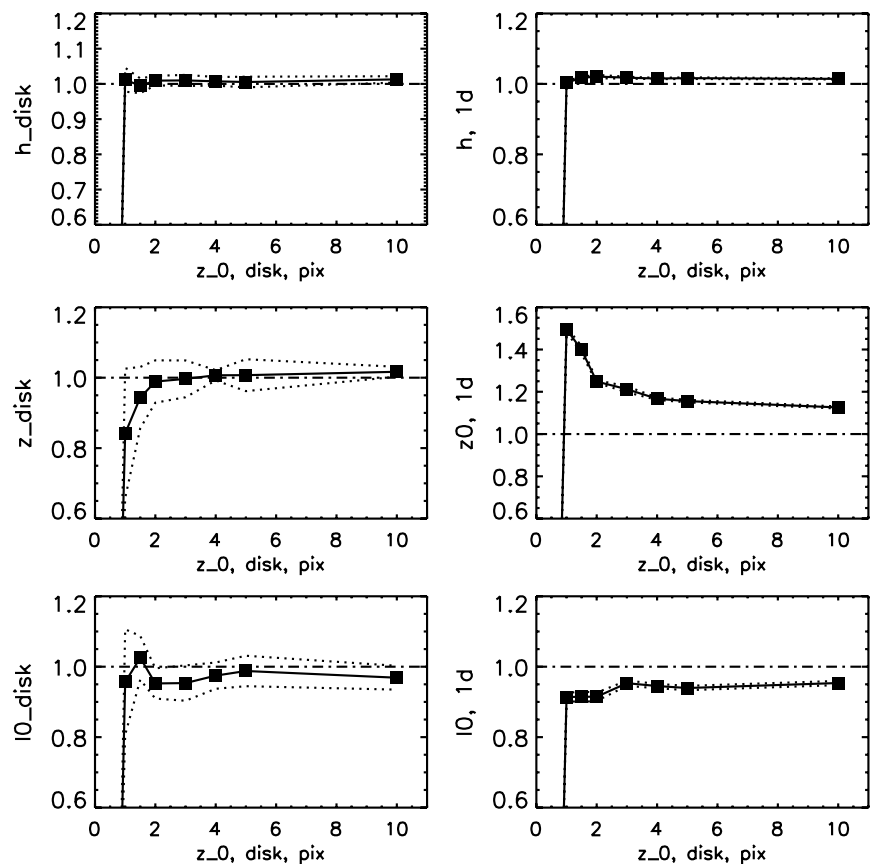

Figure 12. Radial and vertical scales and the central surface brightness (in the arbitrary linear units) estimated from synthetic images in dependence of the vertical scale height expressed in pixels. The left-side panels show results of our 3D analysis, and the right-side panels demonstrate the 1D analysis. The solid curve with symbols shows the averaged output parameters, and the dashed line denotes the input value. The dotted lines designate the $1 \sigma$ uncertainty of the parameters estimated from 21 simulations for each $\mathrm{S} / \mathrm{N}$.

for small galaxies. While deep optical and NIR observations of nearby galaxies allows for seeing very thin and low-contrast disk subsystems as a disk of blue stars reported by SchechtmanRook \& Bershady (2013), most of our galaxies are observed with rather limited resolution (the best SDSS seeing of 1 arcsec

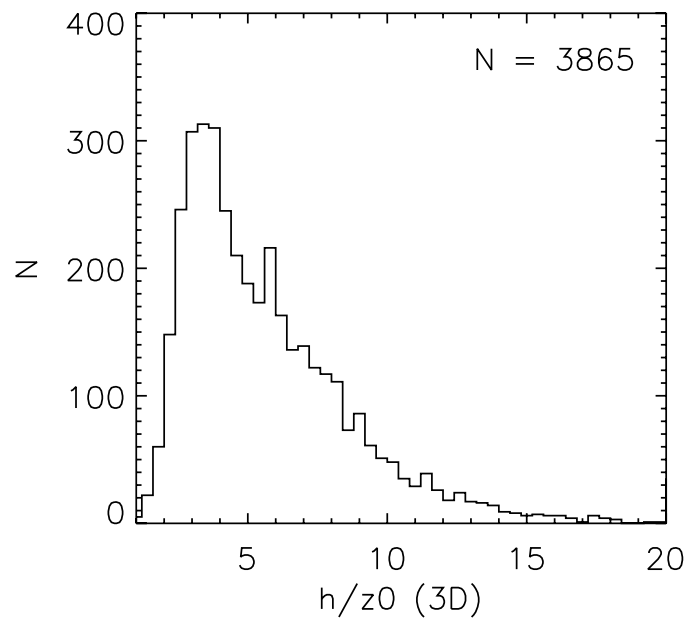

Figure 13. Inverse $r$-band thickness of the stellar disks estimated in our 3D modeling.

corresponds to approximately $1 \mathrm{kpc}$ at $15,000 \mathrm{~km} \mathrm{~s}^{-1}$ ), which makes the study of very thin subsystems impossible with our sample. The same reason prevents us from attempts of selecting the best functional form describing the vertical brightness profiles in thin disks of different galaxies. Figure 17 demonstrates the relationship between our 1D and 3D scale heights. It can be seen that although the trend in Figure 16 can be described as $z_{0,3 \mathrm{D}}=0.58+0.94 \cdot z_{0,1 \mathrm{D}}$, the vertical scales less than $\approx 1$ arcsec deviate significantly from this linear relation. Both the 1D and 3D ways of the scale estimation should be biased for the smallest galaxies in our sample because of the limited angular resolution.

The structural parameters of the stellar disks in the $r$ band determined with our 3D modeling approach are shown in Table 6. 


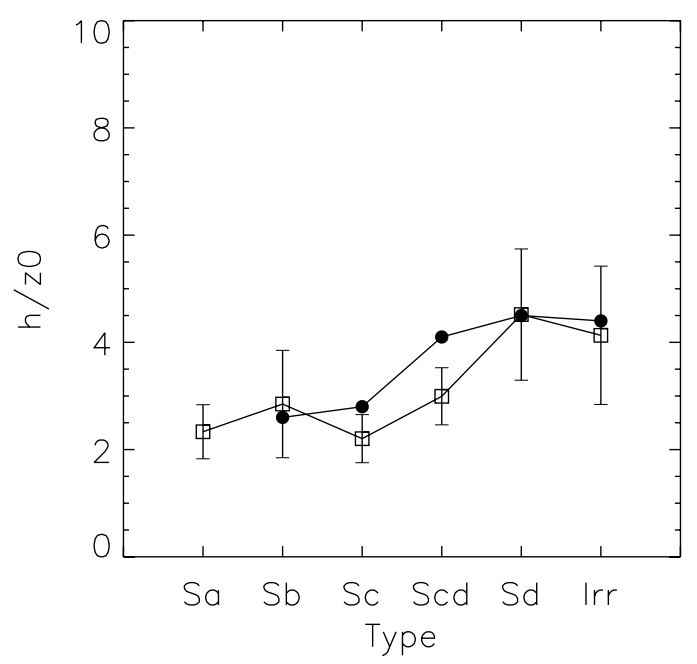

Figure 14. Inverse stellar disk thickness for different morphological types in our sample based on our 1D modeling is shown with the open squares with error bars. The filled circles show the same results by de Grijs (1998).

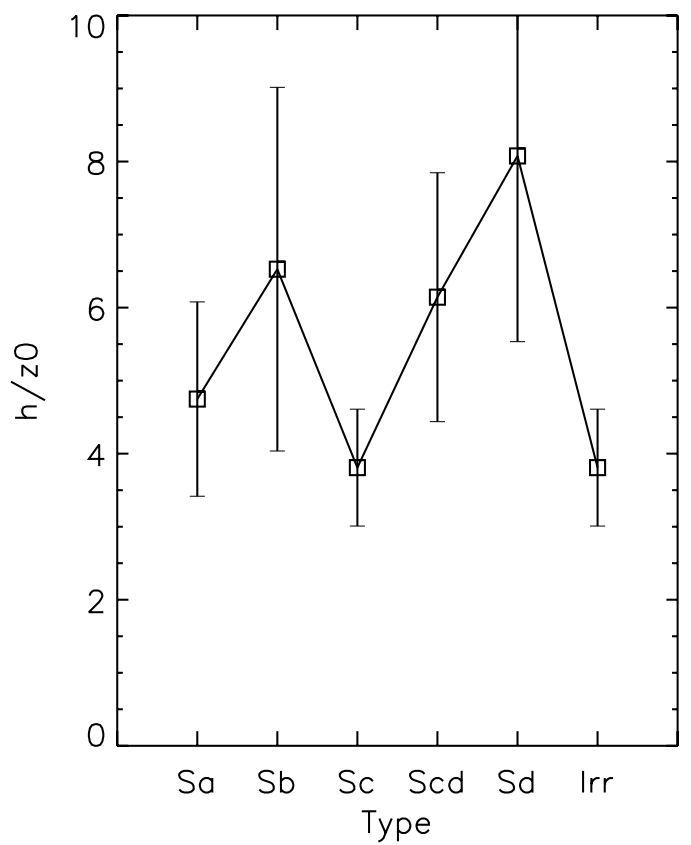

Figure 15. Same as Figure 14 based on our 3D analysis.

Table 6

The Structural Parameters of True Edge-on Galaxies in the $r$ band from our 3D Analysis

\begin{tabular}{lccc}
\hline \hline Name & $h$ & $z_{0}$ & $S_{0}$ \\
\hline EON_113.799_20.000 & 8.86 & 1.04 & 21.34 \\
EON_115.757_45.121 & 6.53 & 2.09 & 19.77 \\
EON_117.764_50.255 & 3.97 & 1.13 & 19.22 \\
EON_119.936_45.366 & 6.77 & 1.54 & 19.46 \\
$\cdots$ & & & \\
\hline
\end{tabular}

Notes. Parameters of the galaxies in the table: EGIS name (same as in Table 4), scale length in arcsec, scale height in arcsec, and face-on central surface brightness in the $r$ band (mag $\operatorname{arcsec}^{-2}$ ).

(This table is available in its entirety in a machine-readable form in the online journal. A portion is shown here for guidance regarding its form and content.)
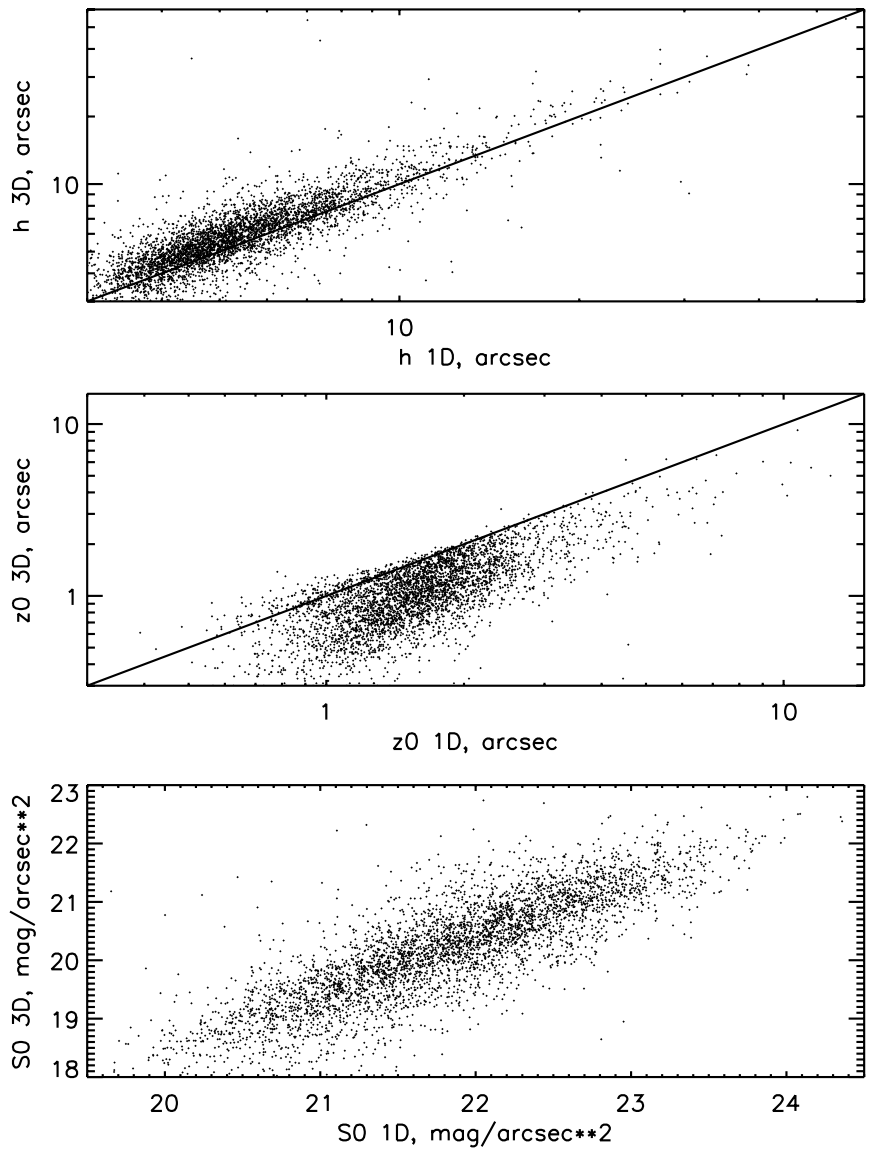

Figure 16. Comparison between the structural parameters of the stellar disks estimated via the 3D modeling and from the $1 \mathrm{D}$ profile analysis. The panels show the radial disk scale length (top), scale height (middle), and the face-on surface brightness (bottom) estimated for the $r$ band images.

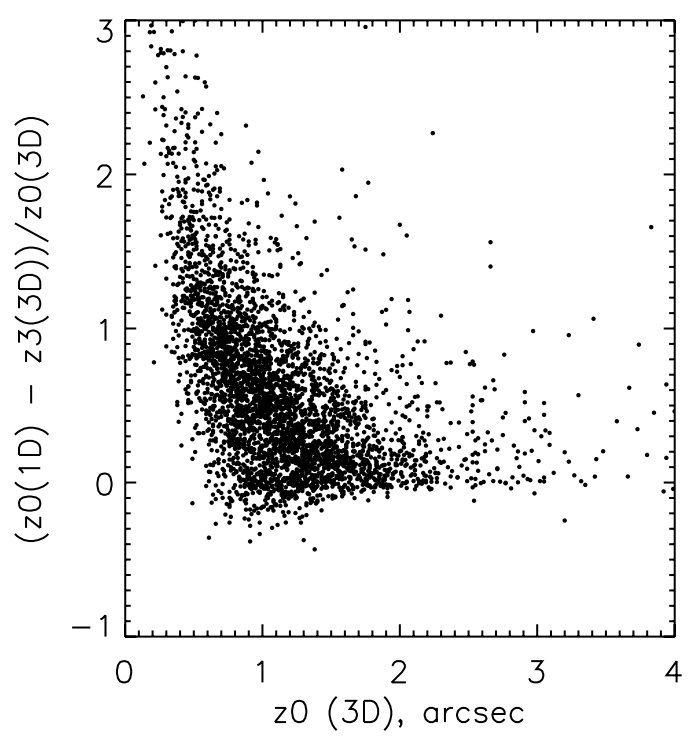

Figure 17. Ratio of the stellar disk thickness estimated via the 3D modeling and from the 1D profile analysis for the $r$ band images. While there is a nearly linear dependence for large values, there is a poor agreement between the scales below 1 arcsec.

Although the amplitude of uncertainties in the $h / z_{0}$ ratio estimated with our 1D approach is less than our typical observing errors, we can estimate the systematic addition to the original $h / z_{0}$ introduced by the non-perfect, edge-on inclination of the 


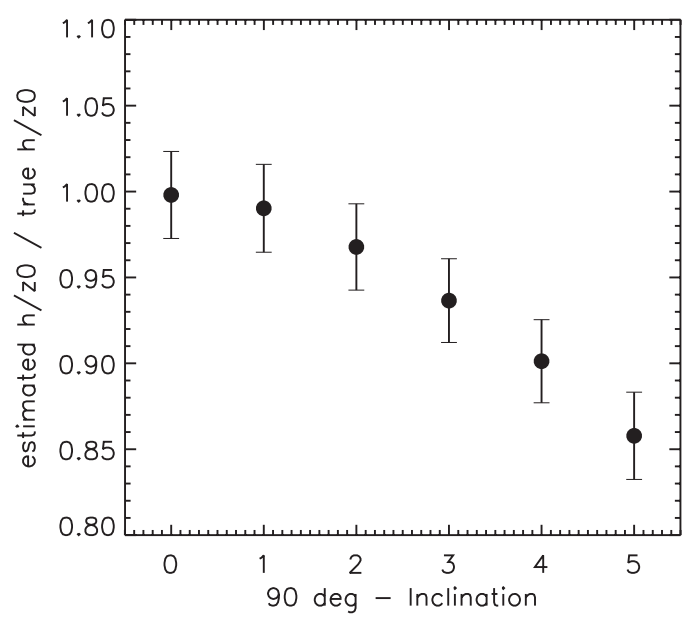

Figure 18. Inverse stellar disk thickness $h / z_{0}$ estimated with our $1 \mathrm{D}$ approach for a set of synthetic images with introduced non-zero inclination of the galactic midplane. The original model $h / z_{0}$ was equal to 4 , and there is no dust in the models. The error bars shown in the picture are 10 times expanded and correspond to the models with central $\mathrm{S} / \mathrm{N}=50$.

galactic midplane to the line of sight. We used the synthetic models developed in Section 4 and estimated the disk thickness $h / z_{0}$ with our 1D approach with respect to the original one introduced to the artificial models. We ran the synthetic images of edge-on galaxies with added noise (corresponding to $\mathrm{S} / \mathrm{N}=$ 50 at the center) through our 1D analysis code. As Figure 18 shows, deviation of the inclination angle from the perfect $90^{\circ}$ introduces a few percent error in the disk thickness estimated with the 1D approach.

\subsection{Limitations to our Estimations of the Structural Parameters}

We have to mention the limitations of our analysis of the galactic structure that originates from the limited angular resolution, relatively low $\mathrm{S} / \mathrm{N}$ of the data, as well as by simplifications assumed for the analysis.

The possibility of a multi-exponential stellar disk is the next step in complication of our 3D modeling, together with introducing disk warps and gradients of the disk scale height. Since this cannot be done for the smallest galaxies in the sample, we will consider possibilities for a more complicated modeling in the next paper.

Including the dust scattering into the modeling requires superior quality optical images, and the analysis remains very uncertain unless a multiwavelength approach is developed (e.g., Baes et al. 2011; Schechtman-Rook et al. 2012). Even in this case, the realistic inclusion of the radiative transfer requires careful evaluation of each object individually. Consideration of more sophisticated shortcuts than plain neglecting the dust scattering and clumpy features in the dust layer in the modeling, which can be applied for our large sample, is a goal of our next work in this direction.

A fundamental limitation to our attempt of separating the galactic structural components based on their different spatial scales comes from the fact that the thickness of the stellar disk in low-massive galaxies is the same as that of the gas disks. As it was noticed by Dalcanton et al. (2004), disk edge-on galaxies with a maximum of the rotation curve less than $120 \mathrm{~km} \mathrm{~s}^{-1}$ do not show regular dust lanes and look mostly clumpy. Including this fact into our modeling requires kinematic information, or at least availability of the distances for all galaxies in the catalog.
We bring consideration of this question out of scope of this paper and notice that our relatively small group, morphologically classified as Irr, may mostly consist of such clumpy galaxies.

Our study does not attempt to provide precision modeling for all galactic components or select the best individual model description to each object from a variety of available models. Instead, it provides a ground for further studies of large samples of edge-on galaxies using both dedicated observations of limited subsamples of certain groups of the galaxies and results from deeper observations that will come from large sky surveys. Incorporating the multiwavelength information and kinematic data for a large fraction of the galaxies in our sample will improve the separation of the structural components.

\section{CONCLUSION}

Careful selection of candidate galaxies from SDSS images allows us to create the largest modern sample (5747 objects) of edge-on galaxies ready for further analysis. Our sample is complete for all galaxies with major axes larger than 30 arcsec. The distribution of the axial ratio shows that our sample size is reasonable and confirms its statistical completeness.

We perform a 1D radial and vertical profile analysis and infer the stellar disk's structural parameters. The results suggest that dust significantly biases the inferred parameters estimated from the optical band images. We also perform a simplified 3D modeling of all our galaxies, taking into account the presence of dust. Comparison between the structural parameters shows that more constrained modeling is needed to eliminate effects of dust in the galaxies.

The catalog can be used for statistical studies of the properties of the thick disks using stacked co-adding images. Our large sample makes possible studying scaling relations for galactic stellar disks and bulges.

We thank AAS Small Research grant program and grants RFBR-11-02-12247, RFBR-11-02-00471, RFBR-12-0200685, and RFBR-14-02-00810 for support. Funding for this project was partly provided through the Nova Southeastern University President's Faculty Research and Development Grant. We thank the anonymous referee whose multiple reviews significantly improved the paper.

Funding for SDSS-III has been provided by the Alfred P. Sloan Foundation, the Participating Institutions, the National Science Foundation, and the US Department of Energy Office of Science.

SDSS-III is managed by the Astrophysical Research Consortium for the Participating Institutions of the SDSS-III Collaboration, including the University of Arizona, the Brazilian Participation Group, Brookhaven National Laboratory, University of Cambridge, Carnegie Mellon University, University of Florida, the French Participation Group, the German Participation Group, Harvard University, the Instituto de Astrofisica de Canarias, the Michigan State/Notre Dame/JINA Participation Group, Johns Hopkins University, Lawrence Berkeley National Laboratory, Max Planck Institute for Astrophysics, Max Planck Institute for Extraterrestrial Physics, New Mexico State University, New York University, Ohio State University, Pennsylvania State University, University of Portsmouth, Princeton University, the Spanish Participation Group, University of Tokyo, University of Utah, Vanderbilt University, University of Virginia, University of Washington, and Yale University.

We acknowledge the usage of the HyperLeda database (http://leda.univ-lyon1.fr). 


\section{REFERENCES}

Abazajian, K. N., Adelman-McCarthy, J. K., Agüeros, M. A., et al. 2009, ApJS, 182,543

Aihara, H., Allende Prieto, C., An, D., et al. 2011, ApJS, 193, 29

Baes, M., Verstappen, J., De Looze, I., et al. 2011, ApJS, 196, 22

Baillard, A., Bertin, E., de Lapparent, V., et al. 2011, A\&A, 532, 74

Barteldrees, A., \& Dettmar, R.-J. 1994, A\&AS, 103, 475

Bergvall, N., Zackrisson, E., \& Caldwell, B. 2010, MNRAS, 405, 2697

Bianchi, S. 2007, A\&A, 471, 765

Bizyaev, D. 2007, BAAS, 39, 758

Bizyaev, D., \& Kajsin, S. 2004, ApJ, 613, 886

Bizyaev, D., \& Mitronova, S. 2002, A\&A, 389, 795

Bizyaev, D., \& Mitronova, S. 2009, ApJ, 702, 1567

Burstein, D. 1979, ApJ, 234, 829

Dalcanton, J. J., Yoachim, P., \& Bernstein, R. A. 2004, ApJ, 608, 189

De Geyter, G., Baes, M., Fritz, J., \& Camps, P. 2013, A\&A, 550, 74

de Grijs, R. 1998, MNRAS, 299, 595

de Grijs, R., Peletier, R., \& van der Kruit, P. 1997, A\&A, 327, 966

de Vaucouleurs, G., de Vaucouleurs, A., Corwin, H. G., Jr., et al. 1991, Third

Reference Catalogue of Bright Galaxies (Volume 1-3, Berlin: Springer)

Fernández Lorenzo, M., Sulentic, J., Verdes-Montenegro, L., et al. 2012, A\&A, 540,47

Giovanelli, R., Haynes, M. P., Salzer, J. J., et al. 1994, AJ, 107, 2036

Hubble, E. 1926, ApJ, 64, 321

Karachentsev, I. D., Karachentseva, V. E., Kudrya, Yu. N., et al. 1999, BSAO, 47,5

Karachentsev, I. D., Karachentseva, V. E., \& Parnovskij, S. L. 1993, AN, 314, 97

Kautsch, S. J. 2009a, AN, 330, 100
Kautsch, S. J. 2009b, PASP, 121, 1297

Kautsch, S. J., Grebel, E. K., Barazza, F. D., \& Gallagher, J. S., III 2006a, A\&A, 445,765

Kautsch, S. J., Grebel, E. K., Barazza, F. D., \& Gallagher, J. S., III 2006b, A\&A, 451,1171

Kormendy, J., \& Bruzual, A. G. 1978, ApJL, 223, L63

Kregel, M., van der Kruit, P., \& de Grijs, R. 2002, MNRAS, 334, 646

Kylafis, N. D., \& Bahcall, J. N. 1987, ApJ, 317, 637

Lintott, C., Schawinski, K., Bamford, S., et al. 2011, MNRAS, 410, 166

Masters, K. L., Nichol, R., Bamford, S., et al. 2010, MNRAS, 404, 792

Matthews, L. D., Gallagher, J. S., \& van Driel, W. 1999, AJ, 118, 2751

Mosenkov, A. V., Sotnikova, N. Ya., \& Reshetnikov, V. P. 2010, MNRAS, 401, 559

Paturel, G., Petit, C., Prugniel, P., et al. 2003, A\&A, 412, 45

Pohlen, M., Balcells, M., Lutticke, R., \& Dettmar, R.-J. 2004, A\&A, 422, 465

Popescu, C. C., Misiriotis, A., Kylafis, N. D., et al. 2000, A\&A, 362, 138

Pranger, F., Böhm, A., Ferrari, C., et al. 2013, A\&A, 557, 62

Schechtman-Rook, A., \& Bershady, M. A. 2013, arXiv:1309.0824

Schechtman-Rook, A., Bershady, M. A., \& Wood, K. 2012, ApJ, 746, 70

Schlegel, D. J., Finkbeiner, D. P., \& Davis, M. 1998, ApJ, 500, 525

Schwarzkopf, U., \& Dettmar, R.-J. 2000, A\&A, 361, 451

Stoughton, C., Lupton, R. H., Bernardi, M., et al. 2002, AJ, 123, 485

Strateva, I., Ivezic, Z., Knapp, G. R., et al. 2001, AJ, 122, 1861

Thuan, T. X., \& Seitzer, P. O. 1979, ApJ, 231, 680

van der Kruit, P. C., \& Searle, L. 1981a, A\&A, 95, 105

van der Kruit, P. C., \& Searle, L. 1981b, A\&A, 95, 116

Xilouris, E. M., Alton, P. B., Davis, J. I., et al. 1998, A\&A, 331, 894

Xilouris, E. M., Byun, Y. I., Kylafis, N. D., et al. 1999, A\&A, 344, 868

Yoachim, P., \& Dalcanton, J. J. 2006, AJ, 131, 226

Zibetti, S., White, S. D. M., \& Brinkmann, J. 2004, MNRAS, 347, 556 